\title{
AVERAGES OF THE COEFFICIENTS OF SCHLICHT FUNCTIONS
}

\author{
G. MILTON WING
}

We shall consider throughout this paper a function

$$
f(z)=\sum_{n=1}^{\infty} a_{n} z^{n}, \quad a_{1}=1,
$$

analytic and schlicht in the unit circle. According to a classical conjecture of Bieberbach, $\left|a_{n}\right| \leqq n$. Recently Bazilevič has proved that $\lim \sup _{n \rightarrow \infty}\left|a_{n}\right| / n \leqq e / 2[1] .{ }^{1}$ Our interest lies in the average behavior of the coefficients. It is clear that if the conjecture holds, then

$$
\left|\sum_{j=1}^{n} a_{j}\right| / C_{n+1,2} \leqq 1 .
$$

More generally, let us define

$$
S_{n}(k)=\sum_{j=0}^{n-1} C_{j+k-1, k-1} a_{n-i} \quad(k \geqq 1)
$$

and

$$
\sigma_{n}(k)=\left|S_{n}(k)\right| / C_{n+k, k+1} .
$$

If $\left|a_{n}\right| \leqq n$, then

$$
\left|S_{n}(k)\right| \leqq \sum_{j=0}^{n-1} C_{j+k-1, k-1}(n-j)=C_{n+k, k+1}
$$

so that $\sigma_{n}(k) \leqq 1$. It is easy to see that the result of Bazilevič implies that

$$
\limsup _{n \rightarrow \infty} \sigma_{n}(k) \leqq \frac{e}{2} .
$$

We prove two theorems concerning the averages $\sigma_{n}(k)$. Using only classical results we obtain a bound on $\lim \sup _{n \rightarrow \infty} \sigma_{n}(k)$ and show that this bound tends to unity for large $k$. By applying recent information concerning the map of the circle $|z|=r<1$ by the function $f(z)$, we get estimates on $\lim \sup _{n \rightarrow \infty} \sigma_{n}(k)$ for small $k$.

Presented to the Society, June 17, 1950; received by the editors August 11, 1950.

${ }^{1}$ Numbers in brackets refer to the bibliography at the end of the paper. 
Theorem 1. Let $k>1$. Then

$$
\limsup _{n \rightarrow \infty} \sigma_{n}(k) \leqq \frac{e^{k+1} \Gamma(k+2) \Gamma(k-1)}{(k+1)^{k+1} 2^{k-1} \Gamma^{2}(k / 2)}=A(k),
$$

and $\lim _{k \rightarrow \infty} A(k)=1$.

Proof. We write

$$
\begin{aligned}
S_{n}(k) & =\frac{1}{2 \pi i} \int_{|z|=r<1} \frac{f(z)}{z^{n+1}(1-z)^{k}} d z \\
& =\frac{1}{2 \pi r^{n}} \int_{0}^{2 \pi} \frac{f\left(r e^{i \theta}\right)}{e^{i n \theta}\left(1-r e^{i \theta}\right)^{k}} d \theta .
\end{aligned}
$$

Hence

$$
\begin{aligned}
\left|S_{n}(k)\right| & \leqq \frac{1}{2 \pi r^{n}} \int_{0}^{2 \pi} \frac{\left|f\left(r e^{i \theta}\right)\right|}{\mid 1-r e^{i \theta \mid k}} d \theta \\
& \leqq \max _{0 \leqq \theta<2 \pi} \frac{\left|f\left(r e^{i \theta}\right)\right|}{2 \pi r^{n}} \int_{0}^{2 \pi} \frac{d \theta}{\left|1-r e^{i \theta}\right|^{k}}
\end{aligned}
$$

By the well known "Distortion Theorem"

$$
\max _{0 \leqq \theta<2 \pi}\left|f\left(r e^{i \theta}\right)\right| \leqq \frac{r}{(1-r)^{2}} \quad(r<1) .
$$

To estimate the integral expression we write

$$
\left|1-r e^{i \theta}\right|=\left(1-2 r \cos \theta+r^{2}\right)^{1 / 2},
$$

so that [2]

$$
\begin{aligned}
\frac{1}{2 \pi} \int_{0}^{2 \pi} \frac{d \theta}{\mid 1-r e^{i \theta \mid k}} & =\frac{1}{2 \pi\left(1-r^{2}\right)^{k / 2}} \int_{0}^{2 \pi} \frac{\left(1-r^{2}\right)^{k / 2}}{\left(1-2 r \cos \theta+r^{2}\right)^{k / 2}} d \theta \\
& =\left(1-r^{2}\right)^{-k / 2} P_{k / 2-1}\left\{\frac{1+r^{2}}{1-r^{2}}\right\} .
\end{aligned}
$$

Here $P_{n(x)}$ is the Legendre function of the first kind of order $n$. Since $\lim _{x \rightarrow \infty} P_{n}(x) / x^{n}=2^{-n} \Gamma(2 n+1) / \Gamma^{2}(n+1)[3$, p. 62], we may write

$$
\left|S_{n}(k)\right| \leqq r^{-n+1}(1-r)^{-2}\left(1-r^{2}\right)^{-k / 2}\left(\frac{1+r^{2}}{1-r^{2}}\right)^{k / 2-1} \phi_{k}(r),
$$

where $\lim _{r \rightarrow 1} \phi_{k}(r)=2^{-k / 2+1} \Gamma(k-1) / \Gamma^{2}(k / 2)$. 
Thus far $r$ has been any number between 0 and 1. We now specify $r=1-(k+1) / n$. Then

$$
\begin{aligned}
\left|\sigma_{n}(k)\right| \leqq & \left(1-\frac{k+1}{n}\right)^{-n+1}\left(\frac{k+1}{n}\right)^{-k-1}(1+r)^{-k / 2} \\
& \cdot\left(\frac{1+r^{2}}{1+r}\right)^{k / 2-1} \phi_{k}(r)\left(C_{n+k, k+1}\right)^{-1} .
\end{aligned}
$$

Since $C_{n+k, k+1} \cong n^{k+1} / \Gamma(k+2)$, we readily compute $\lim \sup _{n \rightarrow \infty} \sigma_{n}(k)$ $\leqq A(k)$, where

$$
A(k)=\frac{e^{k+1} \Gamma(k+2) \Gamma(k-1)}{(k+1)^{k+1} 2^{k-1} \Gamma^{2}(k / 2)} .
$$

That $\lim _{k \rightarrow \infty} A(k)=1$ may now be verified by using Stirling's formula for $\Gamma(k)$.

While the numbers $A(k)$ do tend to unity they decrease very slowly. Computations yield $A(2)=2.23, A(4)=1.42, A(6)=1.26, A(10)$ $=1.15, A(20)=1.07$. Hence even $A(4)$ is greater than $e / 2$. A better estimate of $\lim \sup _{n \rightarrow \infty} \sigma_{n}(k)$ for small $k$ can be obtained by use of the following lemma, recently announced by Bazilevič [1].

LEMMA. The intersection of the circumference $|w|=x, x \geqq r e^{\pi / e}$, with the domain $D(r)$ on which $f(z)$ maps $|z| \leqq r<1$ has linear measure not greater than that of the intersection of the same circumference with the domain $D^{*}(r)$ on which $f^{*}(z)=z /(1-z)^{2}$ maps $|z| \leqq r$.

It follows at once from the lemma that the area $\psi(r)$ of the region $D(r)$ is not greater than $\pi r^{2} e^{2 \pi / e}$ plus the area $\psi^{*}(r)$ of $D^{*}(r)$. Further

$$
\begin{aligned}
\psi^{*}(r) & =\int_{0}^{2 \pi} d \theta \int_{0}^{r} r\left|f^{* \prime}\left(r e^{i \theta}\right)\right|^{2} d r \\
& =\pi \sum_{j=1}^{\infty} j^{3} r^{2 j} \\
& =\frac{\pi r^{2}\left(1+4 r^{2}+r^{4}\right)}{\left(1-r^{2}\right)^{4}} .
\end{aligned}
$$

We may now prove our second theorem.

Theorem 2. Let $k \geqq 1$. Then

$$
\limsup _{n \rightarrow \infty} \sigma_{n}(k) \leqq \frac{k e^{k+1} \Gamma^{1 / 2}(2 k-1)}{(k+1)^{k} 2^{k+1 / 2}}=B(k) .
$$


In particular,

$$
B(1)=1.307, B(2)=1.116, B(3)=1.109 \text {. }
$$

Proof. We apply Schwarz's inequality to (1) to get

(4) $\left|S_{n}(k)\right|^{2} \leqq r^{-2 n}\left\{\frac{1}{2 \pi} \int_{0}^{2 \pi}\left|f\left(r e^{i \theta}\right)\right|^{2} d \theta\right\}\left\{\frac{1}{2 \pi} \int_{0}^{2 \pi} \frac{d \theta}{\left|1-r e^{i \theta}\right|^{2 k}}\right\}$.

To estimate the first integral we write

$$
\begin{aligned}
I & =\frac{1}{2 \pi} \int_{0}^{2 \pi}\left|f\left(r e^{i \theta}\right)\right|^{2} d \theta \\
& =\sum_{j=1}^{\infty}\left|a_{j}\right|^{2} r^{2 j} \\
& =2 \int_{0}^{r} \sum_{j=1}^{\infty} j\left|a_{j}\right|^{2} r^{2 j-1} d r \\
& =\frac{2}{\pi} \int_{0}^{r} \frac{\psi(r)}{r} d r .
\end{aligned}
$$

Hence (2) yields

$$
I \leqq \frac{2}{\pi} \int_{0}^{r}\left\{r \pi e^{2 \pi / e}+\frac{\pi r\left(1+4 r^{2}+r^{4}\right)}{\left(1-r^{2}\right)^{4}}\right\} d r .
$$

An integration by parts then gives

$$
I \leqq \frac{2}{\left(1-r^{2}\right)^{3}}+\frac{g(r)}{(1-r)^{2}},
$$

where $g(r)$ is a function bounded for $0 \leqq r \leqq 1$.

The second integral of (4) can be handled as in Theorem 1. Thus

$$
\left|S_{n}(k)\right|^{2} \leqq r^{-2 n}\left\{\frac{2}{\left(1-r^{2}\right)^{3}}+\frac{g(r)}{(1-r)^{2}}\right\}\left(1-r^{2}\right)^{-k} P_{k-1}\left\{\frac{1+r^{2}}{1-r^{2}}\right\} \text {. }
$$

On choosing

$$
r=1-\frac{k+1}{n}
$$

and carrying out the computations as before, we get the results asserted.

It is interesting to note that for values of $k>3$ the numbers $B(k)$ 
defined in (3) increase, behaving like $2^{-1}\{\pi k\}^{1 / 4}$ for large $k$. The technique of using the Schwarz inequality is thus ineffective for the study of $\lim \sup _{n \rightarrow \infty} \sigma_{n}(k)$ for all but the smaller values of $k$.

\section{BIBLIOGRAPHY}

1. I. E. Bazilevic, On distortion theorems and the coefficients of univalent functions, Doklady Akademii Nauk SSSR (N.S.) vol. 65 (1949) pp. 253-258 (Russian).

2. E. Pinney, On a note of Galbraith and Green, Bull. Amer. Math. Soc. vol. 54 (1948) p. 527.

3. G. Szegö, Orthogonal polynomials, Amer. Math. Soc. Colloquium Publications, vol. $23,1939$.

University of California, Los Angeles 